\title{
NuStar Hard X-Ray View of Low-luminosity Active Galactic Nuclei: High-energy Cutoff and Truncated Thin Disk
}

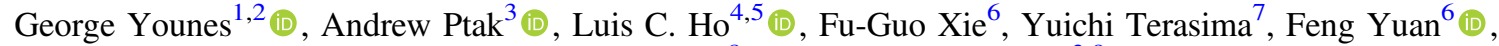 \\ Daniela Huppenkothen $^{8}$ (iD, and Mihoko Yukita ${ }^{3,9}$ \\ ${ }^{1}$ Department of Physics, The George Washington University, Washington, DC 20052, USA; gyounes@gwu.edu \\ ${ }^{2}$ Astronomy, Physics and Statistics Institute of Sciences (APSIS), The George Washington University, Washington, DC 20052, USA \\ ${ }^{3}$ NASA Goddard Space Flight Center, Code 662, Greenbelt, MD 20771, USA \\ ${ }^{4}$ Kavli Institute for Astronomy and Astrophysics, Peking University, Beijing 100871, People's Republic of China \\ ${ }^{5}$ Department of Astronomy, School of Physics, Peking University, Beijing 100871, People's Republic of China \\ ${ }^{6}$ Key Laboratory for Research in Galaxies and Cosmology, Shanghai Astronomical Observatory, Chinese Academy of Sciences, 80 Nandan Road, Shanghai 200030, \\ People's Republic of China \\ ${ }^{7}$ Department of Physics, Ehime University, Bunkyo-cho, Matsuyama, Ehime 790-8577, Japan \\ ${ }^{8}$ Department of Astronomy, University of Washington, 3910 15th Avenue NE, Seattle, WA 98195, USA \\ 9 Johns Hopkins University, Homewood Campus, Baltimore, MD 21218, USA \\ Received 2018 July 30; revised 2018 November 12; accepted 2018 November 21; published 2019 January 10
}

\begin{abstract}
We report the analysis of simultaneous XMM-Newton+Nuclear Spectroscopic Telescope Array (NuSTAR) observations of two low-luminosity active galactic nuclei (LLAGNs), NGC 3998 and NGC 4579. We do not detect any significant variability in either source over the $\sim 3$ day length of the NuSTAR observations. The broadband $0.5-60 \mathrm{keV}$ spectrum of NGC 3998 is best fit with a cutoff power law, while the one for NGC 4579 is best fit with a combination of a hot thermal plasma model, a power law, and a blend of Gaussians to fit an Fe complex observed between 6 and $7 \mathrm{keV}$. Our main spectral results are the following: (1) neither source shows any reflection hump with $3 \sigma$ reflection fraction upper limits of $R<0.3$ and $R<0.18$ for NGC 3998 and NGC 4579 , respectively; (2) the 6-7 keV line complex in NGC 4579 could be fit with either a narrow Fe $\mathrm{K}$ line at $6.4 \mathrm{keV}$ and a moderately broad Fe XXV line or with three relatively narrow lines, which include contribution from Fe XXVI; (3) the NGC 4579 flux is $60 \%$ brighter than previously detected with XMM-Newton, accompanied by a hardening in the spectrum; (4) we measure a cutoff energy $E_{\text {cut }}=107_{-18}^{+27} \mathrm{keV}$ in NGC 3998, which represents the lowest and best constrained high-energy cutoff ever measured for an LLAGN; (5) the NGC 3998 spectrum is consistent with a Comptonization model with either a sphere $(\tau \approx 3 \pm 1)$ or slab $(\tau \approx 1.2 \pm 0.6)$ geometry, corresponding to plasma temperatures between 20 and $150 \mathrm{keV}$. We discuss these results in the context of hard X-ray emission from bright AGNs, other LLAGNs, and hot accretion flow models.
\end{abstract}

Key words: galaxies: active - galaxies: individual (NGC 3998, NGC 4579)

\section{Introduction}

Most galaxies in the nearby universe host a low-luminosity active galactic nucleus (LLAGN), with bolometric luminosities ranging from $10^{38}$ to $10^{43} \mathrm{erg} \mathrm{s}^{-1}$ (Flohic et al. 2006; González-Martín et al. 2009; Zhang et al. 2009). Assuming that the emission is due to accretion onto supermassive black holes with masses $M_{\mathrm{BH}} \approx 10^{7}-10^{9} M_{\odot}$, these bolometric luminosities translate into Eddington ratios in the range $10^{-7}-10^{-3}$, at least an order of magnitude smaller than the ratio for luminous active galactic nuclei (AGNs). The dimness of these LLAGNs is due to an underfed supermassive black hole, and because these sources represent the bulk of active galaxies (Ho et al. 1997), understanding the effect of low accretion rates on the geometry and dynamics of the central engine of galaxies is evidently imperative (see, e.g., Ho 2008, 2009 for reviews).

Multiwavelength observations of LLAGNs have shown a particularly different spectral energy distribution compared to luminous AGNs. For instance, unlike most bright AGNs in the local universe, i.e., Seyfert galaxies, LLAGNs appear as radioloud sources, with radio to X-ray flux ratios comparable to those of radio-loud AGNs. They also lack a UV bump, the ubiquitous feature in almost all AGNs (e.g., Ho 1999; Nagar et al. 2005; Eracleous et al. 2010; Younes et al. 2012).
Specifically, in soft X-rays, LLAGNs do not show the strong, intraday variability shared by their more luminous counterparts (e.g., Ptak et al. 1998; Pian et al. 2010; Younes et al. 2010, 2011; González-Martín \& Vaughan 2012; HernándezGarcía et al. 2014), while their soft X-ray spectra appear mostly featureless, especially lacking the strong reflection features, e.g., a broad $\mathrm{Fe} \mathrm{K} \alpha$ line (Ptak et al. 2004; González-Martín et al. 2009; Younes et al. 2011). Furthermore, LLAGNs do not seem to follow some of the correlations established for typical AGNs; the positive $\Gamma$-Eddington ratio correlation seen in luminous AGNs (Sobolewska \& Papadakis 2009) does not extend down to the limits of LLAGNs. Instead, LLAGNs show an opposite, anticorrelation between the two parameters (Gu \& Cao 2009; Younes et al. 2011; Yang et al. 2015; She et al. 2018). This overwhelming, yet incomplete, list of evidence point toward a drastically altered central engine in LLAGNs compared to luminous AGNs.

These unique properties of LLAGNs cannot be understood in the context of the radiatively efficient, geometrically thin accretion disks that are thought to power luminous AGNs (Shakura \& Sunyaev 1973). For the low accretion rates that govern LLAGNs, the density in the accretion disk becomes too low for radiative cooling to be effective. Hence, the trapped heat will expand the inner parts of the accretion disk into a pressure-supported, radiatively inefficient hot accretion flow 
Table 1

NuSTAR+XMM-Newton Observations of NGC 3998 and NGC 4579

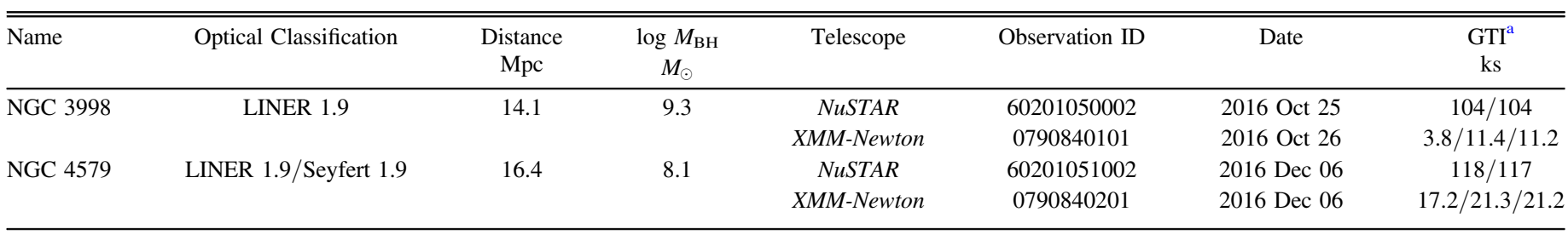

Notes. Optical classification is taken from Ho et al. (1997). Distances are from Tonry et al. (2001) and Springob et al. (2007) for NGC 3998 and NGC 4579, respectively. Black hole masses are calculated using velocity dispersions from Ho et al. (2009) and the $M-\sigma$ relation of Kormendy \& Ho (2013).

${ }^{\mathrm{a}}$ Good time intervals represent the live time of FPMA/FPMB and the cleaned exposure for $\mathrm{pn} / \mathrm{mos} 1 / \mathrm{mos} 2$.

(see, e.g., Narayan \& McClintock 2008; Yuan \& Narayan 2014 for reviews). The most famous examples of such hot accretion solutions are the advection-dominated accretion flow (ADAF; e.g., Narayan \& Yi 1994), the adiabatic inflow-outflow solution (e.g., Blandford \& Begelman 1999), and the convection-dominated accretion flow (Narayan et al. 2000). These models have been successfully fit to the SED of a number of LLAGNs (e.g., Di Matteo et al. 2001; Ptak et al. 2004; Xu \& Cao 2009; Nemmen et al. 2014), including Sgr A* (e.g., Yuan et al. 2004; Wang et al. 2013).

Hard X-ray observations of AGNs are of paramount importance. The X-ray emission of an AGN emanates from the corona, where optical and UV photons from the disk Compton-upscatter into the X-ray band (Haardt \& Maraschi 1993). The temperature in the corona reveals itself through a break in the hard X-ray energies. Moreover, a reflection feature is expected in the hard band in the form of a "Compton hump" at energies of $\sim 30 \mathrm{keV}$. While the brightest AGNs have been studied in the past at those energies (e.g., Mantovani et al. 2016), the Nuclear Spectroscopic Telescope Array $(N u S T A R)$, the first focusing X-ray telescope at hard $\mathrm{X}$-rays, has revolutionized the field, allowing us to study in unprecedented detail those signatures not only in moderately bright AGNs, but for the first time, in LLAGNs as well. Indeed, at least two LLAGNs have been observed with NuSTAR so far, NGC 7213 and M81 (Ursini et al. 2015; Young et al. 2018). Neither source showed any hint of a relativistic disk reflection component in the hard X-ray band, pointing toward a truncated inner accretion disk, possibly filled with a hot accretion flow instead.

Here, we report two deep, simultaneous $N u S T A R+X M M$ Newton observations of the LLAGNs NGC 3998 and NGC 4579. Both galaxies are optically classified as type 1.9 , showing broad $\mathrm{H} \alpha$ emission lines (Table 1; Ho et al. 1997). They have been previously studied in soft X-rays with XMM-Newton (Dewangan et al. 2004; Ptak et al. 2004). Their radio to X-ray SEDs have been successfully fit with an ADAF and/or a jet model (Quataert et al. 1999; Ptak et al. 2004; Xu \& Cao 2009). NGC 3998 and NGC 4579 are among the lowest Eddington ratio AGNs to be observed at hard X-rays $\gtrsim 10 \mathrm{keV}$, with $L_{\mathrm{bol}} / L_{\mathrm{Edd}}$ of $1.0 \times 10^{-5}$ and $1.0 \times 10^{-4}$ for NGC 3998 and NGC 4579, respectively (Younes et al. 2012; Nemmen et al. 2014). Section 2 details the observation and data analysis procedures. We present our timing and spectral results in Section 3, which are discussed in detail in Section 4. Finally, a summary of our findings is presented in Section 5.

\section{Observations and Data Reduction}

The NuSTAR (Harrison et al. 2013) consists of two identical modules, FPMA and FPMB, operating in the energy range 3-79 keV. NuSTAR observed NGC 3998 on 2016 October 25 for a total live-time exposure of $104 \mathrm{ks}$. It observed NGC 4579 on 2016 December 6 for $118 \mathrm{ks}$ (Table 1). We processed the data using the NuSTAR Data Analysis Software, nustardas version v1.8.0 and the calibration files CALDB version number 20180419. We reduced the data using the nuproducts task (which allows for spectral extraction and generation of ancillary and response files) and HEASOFT version 6.22.1. We used the flag saamode=optimized to correct for enhanced background activity visible at the edges of the good time intervals immediately before entering SAA. We extracted source events around the source position using a circular region with $60^{\prime \prime}$ radius, which maximized the signal-to-noise ratio $(\mathrm{S} / \mathrm{N})$. Background events are extracted from an annulus around the source position with inner and outer radii of $120^{\prime \prime}$ and $200^{\prime \prime}$, respectively.

XMM-Newton observed both sources simultaneously with NuSTAR for a total cleaned EPIC-pn exposure of about 4 and 17 ks for NGC 3998 and NGC 4579, respectively (Table 1). During both observations, the EPIC cameras (Strüder et al. 2001) are operated in Full Frame mode, using the thin filter. The PN and MOS data are selected using event patterns $0-4$ and $0-12$, respectively, during only good X-ray events ("FLAG $=0 "$ "). We inspected all observations for intervals of high background, e.g., due to solar flares, and excluded those where the background level was above 5\% of the source flux. We extracted source events for the two observations from a circle with center obtained by running the task eregionanalyse on the cleaned event files. This task calculates the optimal centroid of the count distribution within a given source region. We set the source extraction radius to $60^{\prime \prime}$. Background events are extracted from a source-free annulus centered at the source with inner and outer radii of $120^{\prime \prime}$ and $200^{\prime \prime}$, respectively. We generated response matrix files using the SAS task rmfgen, while ancillary response files are generated using the SAS task arfgen.

We identified two X-ray point sources within 1.5 of NGC 3998 with XMM-Newton. The first, NGC 3998 X-1, is reported in Ptak et al. (2004) as a likely background AGN on the basis of its X-ray, UV, and optical fluxes. The second, NGC 3998 X-2, is an unidentified source. Both sources have very low fluxes, $F_{0.5-10 \mathrm{keV}} \sim 10^{-14} \mathrm{erg} \mathrm{cm}^{-2} \mathrm{~s}^{-1}$. NGC 3998 $\mathrm{X}-1$ is not detected above $2 \mathrm{keV}$, while NGC $3998 \mathrm{X}-2$ is very weakly detected, with a $2-10 \mathrm{keV}$ flux $\lesssim 1 \%$ that of the central 


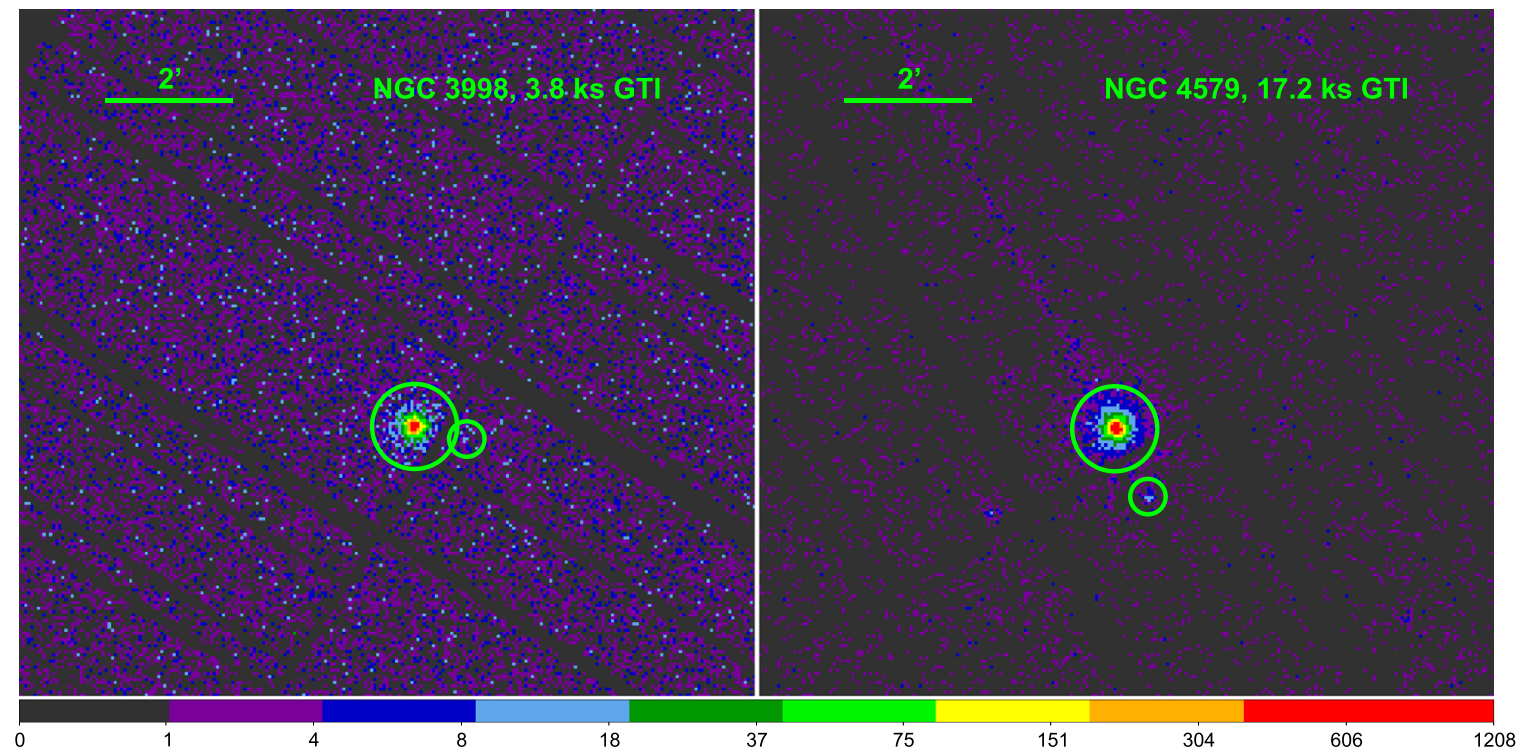

Figure 1. XMM-Newton EPIC-pn image of NGC 3998 (left panel) and NGC 4579 (right panel) in the 2-10 keV energy range. The large green circles, with radii of $60^{\prime \prime}$, are centered at the galactic nuclei. The other circular regions are off-nuclear X-ray point sources. The X-ray emission of these sources is $\lesssim 1 \%$ of the LLAGN. The color bar is in units of counts.
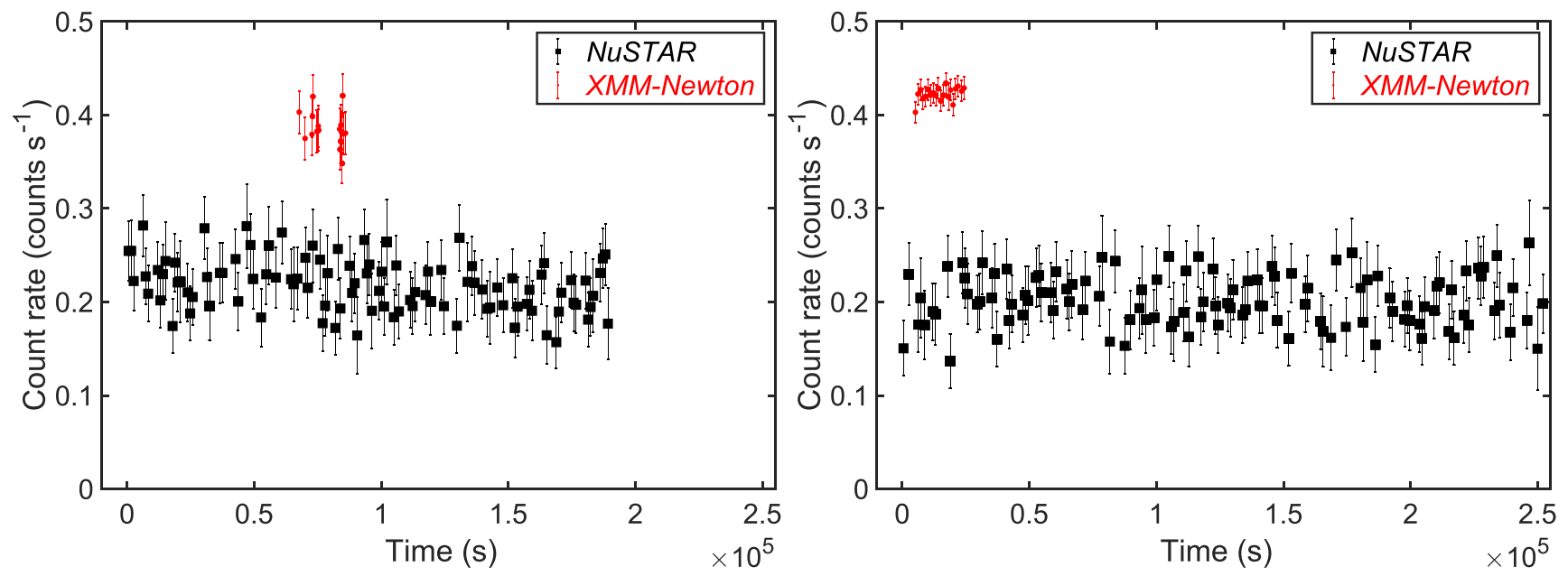

Figure 2. Left panel: NGC 3998 NuSTAR/FPMA and XMM-Newton/PN background-corrected light curves binned at 1 and $0.2 \mathrm{ks}$, in the energy range 3-60 keV and 0.5-10 keV, respectively. Right panel: same for the case of NGC 4579, although both NuSTAR and XMM-Newton light curves are binned to 1 ks. For clarity, the XMM-Newton rate has been scaled down by a factor of 8 and 7 for NGC 3998 and NGC 4579, respectively. In both panels, time 0 represents the start time of the NUSTAR observation.

LLAGN (Figure 1, left panel). We also detect two weak X-ray point sources in the vicinity of NGC 4579 . Only one is detected $>2 \mathrm{keV}$, with a flux that is $\sim 1 \%$ compared to the $2-10 \mathrm{keV}$ flux of the nucleus (Figure 1, right panel). We conclude that neither LLAGN as observed with NUSTAR is contaminated by extranuclear point sources.

The spectral analysis of the NUSTAR and XMM-Newton data was performed using Xspec version 12.9.1p (Arnaud 1996). The photoelectric cross-sections of Verner et al. (1996) and the abundances of Wilms et al. (2000) are used throughout to account for absorption by neutral gas. We bin the spectra to have an $\mathrm{S} / \mathrm{N}$ of 6 in each spectral bin and used the $\chi^{2}$ statistic in Xspec for model parameter estimation and error calculation. For all spectral fits, we added a multiplicative constant normalization between FPMA and FPMB, frozen to 1 for the former and allowed to vary for the latter to account for any calibration uncertainties between the two instruments. We applied the same strategy to account for any calibration uncertainties between the different EPIC instruments. We find these calibration uncertainties to be within $2 \%$. We also allowed for a constant normalization factor between NuSTAR and XMM-Newton instruments and found that such an uncertainty is around $\sim 10 \%$. Finally, all quoted errors throughout the manuscript are at the $1 \sigma$ level, unless otherwise noted.

\section{Results}

\subsection{Timing Analysis}

Figure 2 shows the background-corrected NUSTAR and $X M M$-Newton light curves, in the energy range $3-60 \mathrm{keV}$ and $0.5-10 \mathrm{keV}$, respectively, for both NGC 3998 and NGC 4579. All light curves are binned at $1 \mathrm{ks}$ resolution, except for the XMM-Newton observation of NGC 3998. It is binned at $200 \mathrm{~s}$ resolution to accommodate the many short good time intervals as a result of filtering out time intervals of high background 

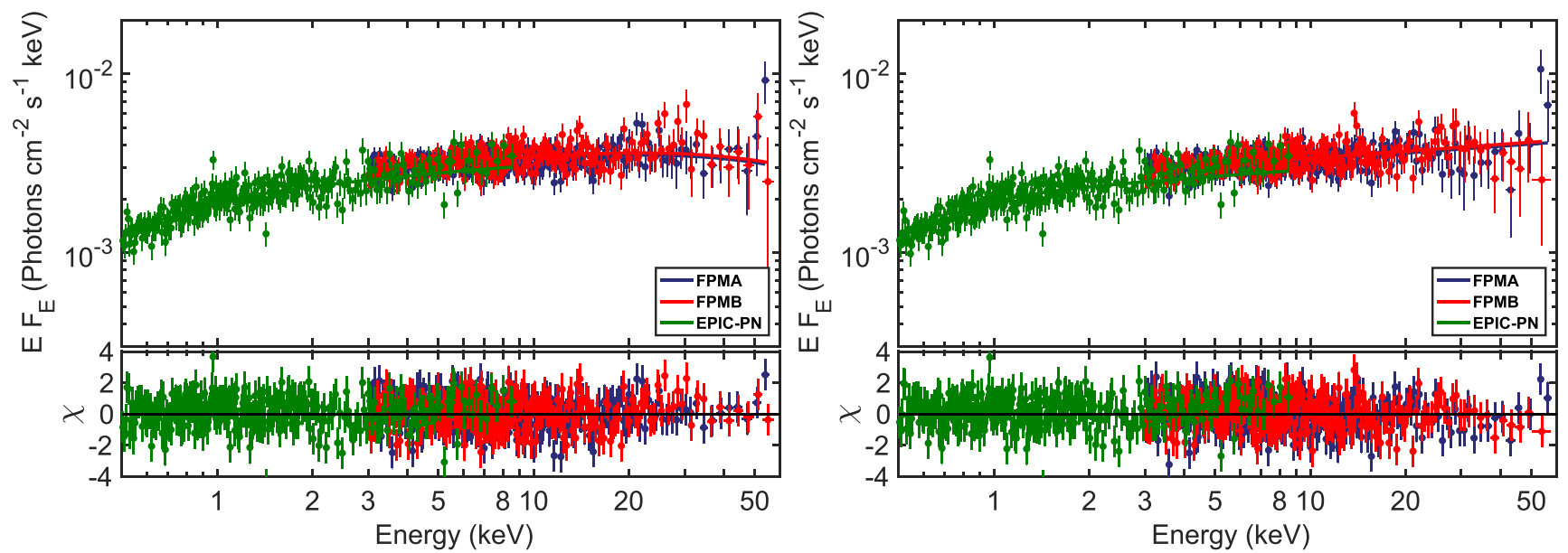

Figure 3. Upper-left panel: best-fit cutoff PL model (solid line) to the NuSTAR+XMM-Newton spectra of NGC 3998 (filled circles; only EPIC-pn plotted for clarity) shown in $E F_{\mathrm{E}}$ space. Upper-right panel: best-fit PL model (solid line) to the NuSTAR+XMM-Newton spectra of NGC 3998 (filled circles; only EPIC-pn plotted for clarity) shown in $E F_{\mathrm{E}}$ space. Lower panels: residuals in terms of the standard deviation $\sigma$. See text for more details.

Table 2

NGC 3998 Best-fit Spectral Parameters

\begin{tabular}{|c|c|c|c|c|c|}
\hline NGC 3998 & Cutoff PL & pexrav & pexmon & compTT(slab) & compTT(sphere) \\
\hline$N_{\mathrm{H}}\left(10^{22} \mathrm{~cm}^{-2}\right)$ & $0.042 \pm 0.005$ & $0.045 \pm 0.005$ & $0.047 \pm 0.006$ & $0.045 \pm 0.005$ & $0.044 \pm 0.005$ \\
\hline$k T(\mathrm{keV})$ & $\ldots$ & $\ldots$ & $\ldots$ & $38_{-11}^{+22}$ & $32_{-8}^{+19}$ \\
\hline$\tau$ & $\ldots$ & $\cdots$ & $\cdots$ & $1.2 \pm 0.6$ & $3 \pm 1$ \\
\hline$\Gamma$ & $1.79 \pm 0.01$ & $1.80 \pm 0.01$ & $1.80 \pm 0.02$ & $\ldots$ & $\ldots$ \\
\hline$E_{\text {cutoff }}(\mathrm{keV})$ & $107_{-18}^{+27}$ & $111_{-23}^{+38}$ & $104_{-22}^{+39}$ & $\cdots$ & $\cdots$ \\
\hline$R$ & $\ldots$ & $<0.06$ & $<0.09$ & $\ldots$ & $\ldots$ \\
\hline$F_{0.5-10 \mathrm{keV}}\left(10^{-11} \mathrm{erg} \mathrm{s}^{-1} \mathrm{~cm}^{-2}\right)$ & $1.17 \pm 0.01$ & $1.16 \pm 0.01$ & $1.16 \pm 0.02$ & $1.17 \pm 0.01$ & $1.20 \pm 0.01$ \\
\hline$\overline{\chi^{2} / \text { dof }}$ & $1217 / 1221$ & $1218 / 1220$ & $1217 / 1220$ & $1218 / 1221$ & $1218 / 1221$ \\
\hline
\end{tabular}

flaring activity. Nonetheless, we find no obvious variability in either source over the full length of the XMM-Newton and NuSTAR observations, the latter spanning $\gtrsim 2.5$ days in length. Using XMM-Newton, we derive a $3 \sigma$ upper limit of $\sim 10 \%$ on the flux variability on timescales of $1 \mathrm{ks}$. On longer timescales of 5 and $10 \mathrm{ks}$, we derive $3 \sigma$ upper limits of $\sim 30 \%$ and $\sim 20 \%$ on the flux variability using NuSTAR. For a more rigorous look at the variability of both sources, we built the power spectral density (PSD) using the Lomb-Scargle periodogram and NuSTAR light curves binned at $60 \mathrm{~s}$. The PSD of the two sources consist of only white noise with no significant red noise component in the frequency range $\sim 10^{-5}-0.017 \mathrm{~Hz}$.

\subsection{Spectral Analysis}

\subsubsection{NGC 3998}

Before we delved into the spectral analysis of the NuSTAR$+X M M$-Newton observations, we first checked the highest energy bin in which NGC 3998 is detected with NuSTAR. We calculated the number of exposure and background-corrected counts from the source location at energies $>10 \mathrm{keV}$, in energy bins of $10 \mathrm{keV}$. We detect the source in the energy bin $50-60 \mathrm{keV}$ with a significance of $3.4 \sigma, 58 \pm 17$ counts. Above $60 \mathrm{keV}$, the source becomes indistinguishable from the background. Hence, all spectral analysis for NGC 3998 was done in the $3-60 \mathrm{keV}$ range for NuSTAR and $0.5-10 \mathrm{keV}$ for XMMNewton.
We started our spectral analysis with a simple absorbed (using tbabs model in Xspec) power-law (PL) fit to the NuSTAR+ $X M M$-Newton spectra simultaneously. This simple model is the preferred one to fit the previous XMM-Newton-only data (e.g., Ptak et al. 2004). We find a statistically acceptable fit with a $\chi^{2}$ of 1244 for 1222 degrees of freedom (dof). We find a small intrinsic hydrogen column density of about $4 \times 10^{20} \mathrm{~cm}^{-2}$ and a hard photon index $\Gamma=1.86 \pm 0.01$. Nevertheless, structured residuals are visible mainly at high energies (Figure 3). Hence, we replaced the PL with a high-energy exponentially cutoff one- the cutoffpl model in Xspec. The reduced $\chi^{2}$ for this fit is 1217 for 1221 dof. This is an improvement of $\Delta \chi^{2}=27$ for one additional free parameter, the cutoff energy. An F-test comparison between the two models indicates that the probability for the improvement due to the cutoffpl fit (compared to the one with a single absorbed PL) to occur by chance is $2.4 \times 10^{-7}$. We conclude that the cutoff energy is required by the data and that the cutoffpl model is the best-fit model to the NuSTAR+XMM-Newton spectra of NGC 3998.

The cutoff energy is well constrained by our data $E_{\text {cut }}=$ $107_{-18}^{+27} \mathrm{keV}$, and the photon index is slightly harder, $\Gamma=1.79 \pm 0.01$, compared to the PL model. We find a $0.5-10 \mathrm{keV}$ flux of about $(1.17 \pm 0.01) \times 10^{-11} \mathrm{erg} \mathrm{s}^{-1} \mathrm{~cm}^{-2}$ and a slightly lower one, $0.97_{-0.02}^{+0.03} \times 10^{-11} \mathrm{erg} \mathrm{s}^{-1} \mathrm{~cm}^{-2}$, in the hard $10-60 \mathrm{keV}$ band. The $0.5-10 \mathrm{keV}$ flux that we measure is consistent, within errors, with the one measured 15 years earlier with XMM-Newton (Ptak et al. 2004; Younes et al. 2011). The 


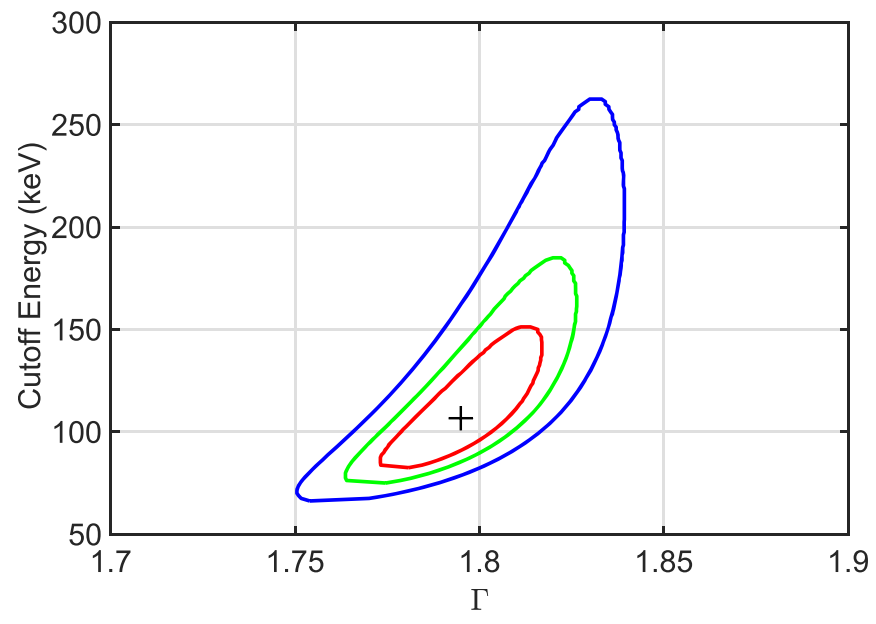

Figure 4. NGC 3998 contour plot of the cutoff energy and PL index $\Gamma$; red, green, and blue show the $1 \sigma, 2 \sigma$, and $3 \sigma$ contours, respectively.

best-fit spectral parameters are summarized in Table 2, while the data and best-fit cutoff PL model are shown in Figure 3. We also show in Figure 4 the $1 \sigma, 2 \sigma$, and $3 \sigma$ contours of the cutoff energy $E_{\text {cut }}$ and the photon index $\Gamma$.

Although we detect no sign of reflection off of dense material, e.g., from an accretion disk or a dense molecular torus, we fit the NuSTAR+XMM-Newton spectra with a reflection model to assess the reflection fraction limit that we can derive with our data. We used the pexrav model implemented in Xspec, which utilizes as continuum an exponentially cut off PL (Magdziarz \& Zdziarski 1995). The model is also dependent on the inclination angle of the source, which we fixed to $45^{\circ}$, and on elemental abundances which we assumed to be solar (we verified that changing these parameters within a valid range does not affect our results). We find a good fit to the data with this model with $\chi^{2}=1218$ for 1220 dof. The result is consistent with no reflection, and we derive a $1 \sigma$ upper limit on the reflection fraction of a fiducial reflection component of 0.06. The continuum fit parameters are fully consistent with that of the cutoffpl model fit. We also fit the data using the pexmon model instead of pexrav, which has the advantage of self-consistently including reflection due to atomic species such as the $\mathrm{Fe} \mathrm{K} \alpha, \mathrm{Fe} \mathrm{K} \beta$, and $\mathrm{Ni} \mathrm{K} \alpha$ (Nandra et al. 2007). We find a statistically equivalent fit with $\chi^{2}=1217$ for 1220 dof. The only noticeable difference between the two models is the constraint on the reflection fraction, which is shown as contour plots in Figure 5. Due to the non-detection of any $\mathrm{Fe}$ lines in the spectrum of NGC 3998, the allowed parameter space for the reflection fraction in the case of pexmon is more stringent. Hence, we consider as conservative the upper limit on $R$ as derived with pexrav. The constraint on the cutoff energy is similar in the two cases. The best-fit parameters of both models are listed in Table 2.

Finally, we fit the NGC 3998 spectrum with physically motivated emission models. Assuming that the accretion geometry is consistent with a hot flow, the emission process is expected to be either thermal bremsstrahlung and/or Comptonization of soft photons by the hot plasma in the flow (see Yuan \& Narayan 2014 and references therein). Hence, we fit the spectrum with the thermal bremsstrahlung model zbrems in Xspec. This model does not give a good fit to the data with a $\chi^{2}$ of 2898 for 1222 dof. Fitting the data with a model consisting of two thermal bremsstrahlung model gives a $\chi^{2}$ of 1294 for 1219 dof, considerably worse than the cutoff PL model and resulting in strong residuals at hard X-rays. We next fit the spectrum with the Comptonization model compTT in Xspec (Titarchuk 1994). We assumed that the seed photon temperature is $10 \mathrm{eV}$. Assuming either a spherical or slab geometry for the Compton cloud, we get a good fit to the data with a $\chi^{2}$ of 1218 for 1222 dof, equivalent in goodness to the phenomenological cutoff PL model. In the spherical geometry case, we get a plasma temperature $k T=32_{-8}^{+19} \mathrm{keV}$ and an optical depth $\tau=3 \pm 1$, while the slab geometry results in a plasma temperature $k T=38_{-11}^{+22} \mathrm{keV}$ and an optical depth $\tau=1.2 \pm 0.6$. The contour plots of the optical depth and temperature are shown in Figure 6 for the spherical (dashed lines) and the slab (solid lines) geometries, respectively. Regardless of the geometry assumed, we find a similar plasma temperature in the range $15-150 \mathrm{keV}$. The optical depth, on the other hand, is a factor of 2-3 smaller for the slab geometry compared to the spherical case. We discuss these results in Section 4.

\subsection{2. $N G C 4579$}

Similar to our previous analysis, we first determined the highest energies at which NGC 4579 is detected with NuSTAR. Although the background was slightly larger in this case, we detect NGC 4579 up to $60 \mathrm{keV}$ at the $\sim 3 \sigma$ level, with a background- and exposure-corrected number of counts of $51 \pm 18$.

We fit the NGC $4579 N u S T A R+X M M-N e w t o n$ spectra simultaneously, starting with a simple absorbed PL. The fit is statistically poor, with a $\chi^{2}$ of 2312 for 1840 dof. Residuals due to this fit were clear at low energies and in the form of emission lines around the Fe-line complex. Hence, we decided to ignore data in the energy range $5.5-8.0 \mathrm{keV}$ in order to first establish the best-fit continuum model. We note that the intrinsic absorption to NGC 4579 was consistent with 0 with a $3 \sigma$ upper limit of $0.004 \times 10^{22} \mathrm{~cm}^{-2}$; therefore, in the following we removed the contribution from any intrinsic absorber. Most early-type galaxies emit an extended hot diffuse X-ray component usually fit to an emission model from an optically thin plasma (Fabbiano 1989). Hence, we fit the broadband spectrum of NGC 4579 with a combination of a PL and the Xspec mekal component (Mewe et al. 1985; Kaastra \& Mewe 1993) to model the thermal emission from hot diffuse gas in the soft band. The fit is statistically good with a $\chi^{2}$ of 1759 for 1675 dof, and no strong residuals are present at low energies. We find a plasma temperature $k T=0.63 \pm 0.02$ and a PL photon index $\Gamma=1.822 \pm 0.006$. Replacing the PL model with a cutoff PL, we find an equally good fit with a $\chi^{2}$ of 1756 for 1674 dof. Hence, the data do not statistically require a high-energy cutoff. We place a $3 \sigma$ upper limit on any fiducial cutoff $E_{\text {cut }}>152 \mathrm{keV}$. For consistency with NGC 3998, in the following we utilize the cutoff PL model to establish best-fit parameters for the Fe-line complex. None of the fit parameters are affected if we use a simple PL model.

The residuals that we observe in our data around the Fe complex cover the full $\sim 6$ to $\sim 7 \mathrm{keV}$ energy range (Figure 7). Previously, these residuals have been fit with either a narrow emission line at $\sim 6.4 \mathrm{keV}$ and a broad component at slightly higher energies (Dewangan et al. 2004), or a combination of three narrow lines at energies fixed at the $\mathrm{Fe} \mathrm{K}$ and the highly ionized Fe species, Fe XXV and Fe XXVI 

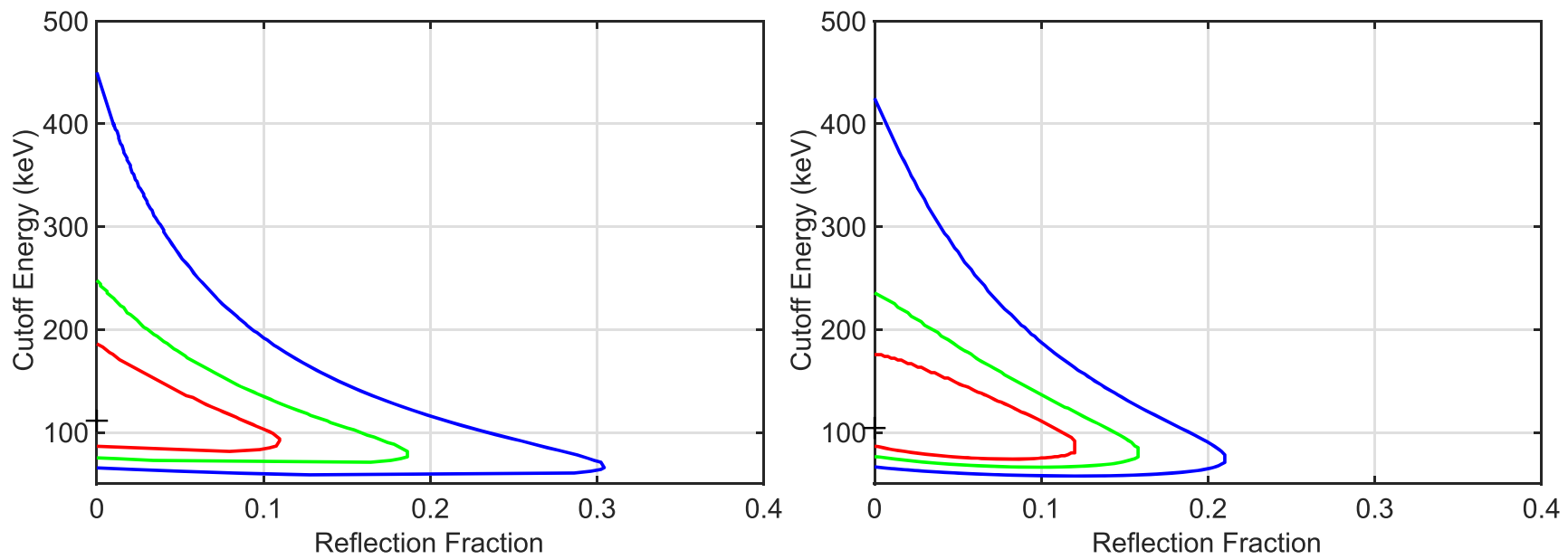

Figure 5. Contour plot of the high-energy cutoff and reflection fraction from optically thick material, $R$. The left panel shows the results using the pexrav model, while the right panel shows the effect of using the pexmon model, which self-consistently includes reflection from atomic species. The red, green, and blue lines show the $1 \sigma, 2 \sigma$, and $3 \sigma$ contours, respectively.

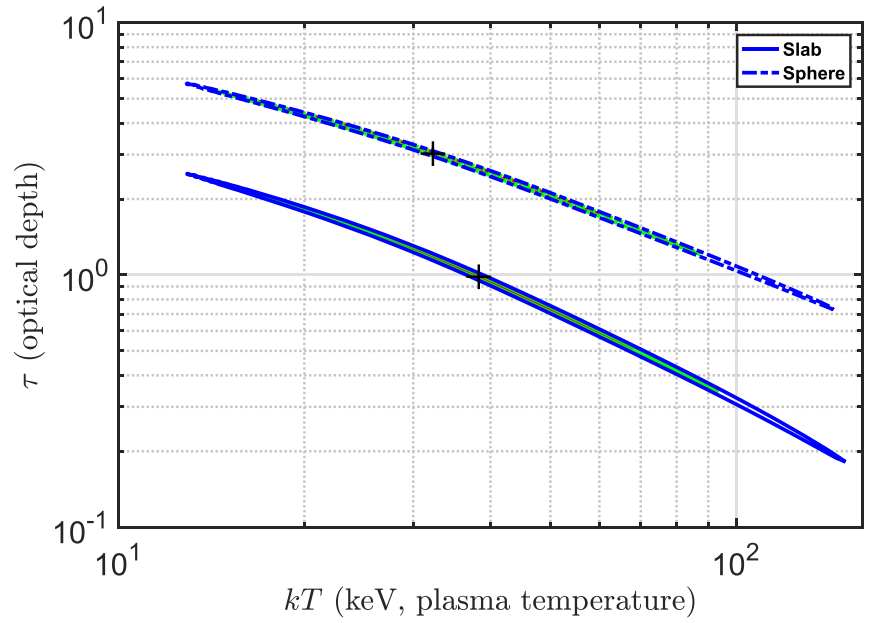

Figure 6. Contour plot of the plasma temperature and optical depth for the compTT model fit to the NGC 3998 data, assuming a slab geometry (solid lines) and spherical geometry (dashed lines); the red, green, and blue lines show the $1 \sigma, 2 \sigma$, and $3 \sigma$ contours, respectively.

(Terashima et al. 1998). Hence, we first added to our continuum model two Gaussian line profiles. These two components eliminate any outstanding residuals in the energy range $6-7 \mathrm{keV}$, and we find a $\chi^{2}$ of about 1955 for 1880 dof for the full $0.5-60 \mathrm{keV}$ fit. We indeed find that the two lines consist of a narrow component at $6.4 \pm 0.1 \mathrm{keV}$ with an unresolved width (fixed to its best-fit value of $0.018 \mathrm{keV}$ ) and a broader component with energy centered at $6.6 \pm 0.1 \mathrm{keV}$, consistent with emission from highly ionized Fe XXV. Second, we fit the Fe complex with three Gaussian components with energies fixed at 6.4, 6.7, and $6.97 \mathrm{keV}$. Without fixing the widths of the lines, there is no unique solution and there is a tendency for Fe XXV or Fe XXVI to acquire large widths $(\sim 3.2 \mathrm{keV})$ and fit part of the continuum. Hence, to test this hypothesis, we also fixed the widths of these lines to 0 , i.e., assuming narrow components. We find an equally good fit with a $\chi^{2}$ of 1970 for 1882 dof.

Figure 8 shows the broadband best-fit model and a zoom-in at the Fe-line complex fit with two Gaussian components (the residuals due to a fit with three narrow Gaussians look fairly

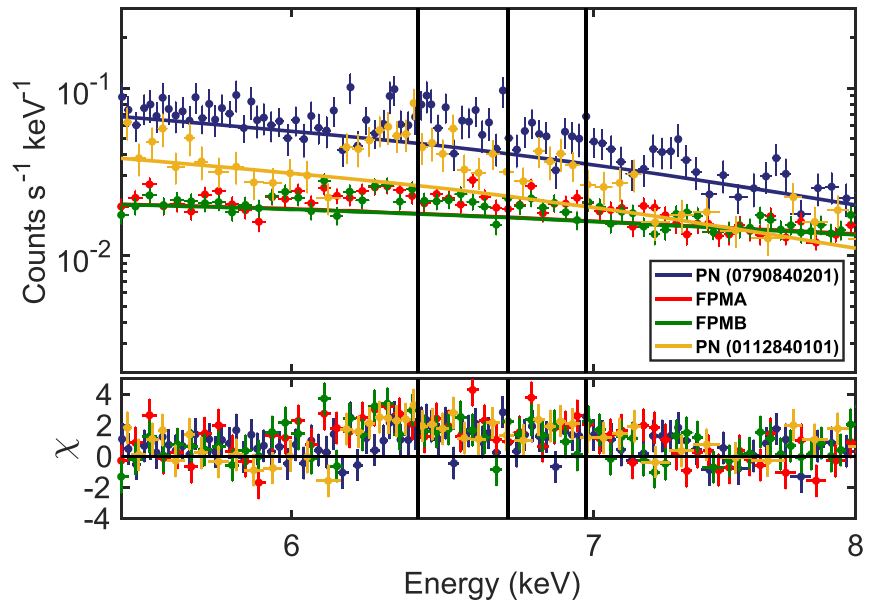

Figure 7. Upper panel: count spectrum of our current NuSTAR+XMM-Newton observation (red, green, and blue dots, for FPMA, FPMB, and pn, respectively) and the previous XMM-Newton-only observation (pn, yellow dots), zoomed-in at the Fe complex. The solid lines are the best-fit continuum to all data simultaneously excluding the $5.5-8.0 \mathrm{keV}$ energy range. The vertical black lines are plotted at the expected energies of the $\mathrm{Fe} \mathrm{K}(6.4 \mathrm{keV}), \mathrm{Fe} \mathrm{XXV}$ $(6.7 \mathrm{keV})$, and Fe XXVI (6.97 keV) lines. Lower panel: residuals in terms of the standard deviation $\sigma$. Notice that the residuals deviate from the best-fit model in the full 6.0-7.0 energy range, indicating an Fe complex consisting of multiple ionization species. See the text for more details.

similar). We report the best-fit model parameters of the continuum in Table 3, while the Fe lines' best-fit parameters are reported in Table 4. Figure 9 shows the $1 \sigma, 2 \sigma$, and $3 \sigma$ contours of $E_{\text {cut }}$ and $\Gamma$.

Similar to NGC 3998, we do not detect any sign of reflection off of a geometrically thick accretion disk. Nonetheless, in order to establish an upper limit on any fiducial reflection component, we replace the cutoff PL with the pexrav model in fitting the broadband spectrum of NGC 4579. We fixed the inclination angle of the disk to $45^{\circ}$ and assumed solar abundances. We find a good fit to the data with a $\chi^{2}=1953$ for 1880 dof. We find a $1 \sigma$ upper limit on the reflection $R \lesssim 0.03$. The continuum fit parameters are fully consistent with that of the cutoffpl model fit. The best-fit parameters are listed in Table 2, and we show in Figure 9 the $1 \sigma, 2 \sigma$, and $3 \sigma$ contours of $R$ and $E_{\text {cut }}$. These results are discussed in Section 4. 

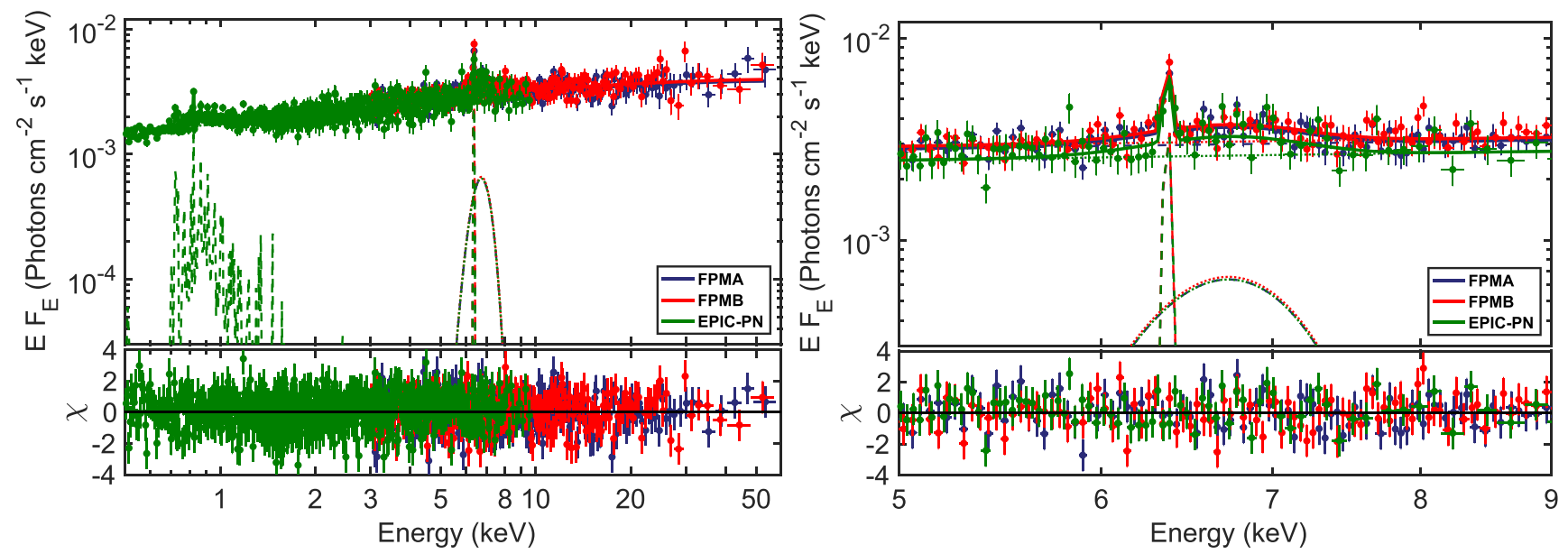

Figure 8. Upper panels: best-fit model to the NuSTAR+XMM-Newton spectra of NGC 4579 (only EPIC-pn shown for clarity). The model consists of a cutoff PL, emission from a hot thermal plasma, and two Gaussian lines. The different components are shown as dashed lines while the sum of all is shown as a solid line. The left panel shows the broadband spectrum while a zoom-in at the Fe complex is shown in the right panel. Lower panels: residuals in terms of the standard deviation $\sigma$. See the text for more details.

Table 3

NGC 4579 Best-fit Continuum Parameters

\begin{tabular}{|c|c|c|c|c|c|}
\hline NGC 4579 & mekal+cutoffpl & mekal+pexrav & mekal+pexmon & mekal+compTT(slab) & mekal+compTT(sphere) \\
\hline$k T(\mathrm{keV}, \mathrm{compTT})$ & $\ldots$ & $\ldots$ & $\ldots$ & $>166$ & $>179$ \\
\hline$\tau$ & $\cdots$ & $\cdots$ & $\cdots$ & $0.05_{-0.02}^{+0.10}$ & $0.31_{-0.16}^{+0.41}$ \\
\hline$k T$ (keV, mekal) & $0.63 \pm 0.02$ & $0.63 \pm 0.02$ & $0.62 \pm 0.02$ & $0.62 \pm 0.02$ & $0.62 \pm 0.02$ \\
\hline \multirow[t]{2}{*}{$\Gamma$} & $1.81 \pm 0.01$ & $1.80 \pm 0.01$ & $1.81 \pm 0.01$ & $\cdots$ & $\cdots$ \\
\hline & $1.87 \pm 0.01^{\mathrm{a}}$ & $\cdots$ & $\cdots$ & $\cdots$ & $\cdots$ \\
\hline$E_{\text {cutoff }}(\mathrm{keV})$ & $>299$ & $409_{-152}^{+583}$ & $414_{-158}^{+146}$ & $\cdots$ & $\cdots$ \\
\hline$R$ & $\cdots$ & $<0.03$ & $<0.02$ & $\cdots$ & $\cdots$ \\
\hline \multirow{2}{*}{$F_{0.5-10 \mathrm{keV}}\left(10^{-11} \mathrm{erg} \mathrm{s}^{-1} \mathrm{~cm}^{-2}\right)$} & $1.08 \pm 0.01$ & $1.08 \pm 0.01$ & $1.07 \pm 0.01$ & $1.07 \pm 0.01$ & $1.08 \pm 0.01$ \\
\hline & $0.652 \pm 0.005^{\mathrm{a}}$ & $\ldots$ & $\ldots$ & $\ldots$ & - \\
\hline$F_{10-60 \mathrm{keV}}\left(10^{-11} \mathrm{erg} \mathrm{s}^{-1} \mathrm{~cm}^{-2}\right)$ & $1.04 \pm 0.02$ & $1.03 \pm 0.02$ & $1.03 \pm 0.02$ & $1.02 \pm 0.02$ & $1.03 \pm 0.02$ \\
\hline$L_{0.5-60 \mathrm{keV}}\left(10^{41} \mathrm{erg} \mathrm{s}^{-1}\right)$ & $6.8 \pm 0.1$ & $6.8 \pm 0.1$ & $6.7 \pm 0.1$ & $6.7 \pm 0.1$ & $6.8 \pm 0.1$ \\
\hline$\chi^{2} /$ dof & $1955 / 1880$ & $1953 / 1880$ & $1954 / 1880$ & $1955 / 1880$ & $1955 / 1880$ \\
\hline
\end{tabular}

Note.

${ }^{\text {a }}$ Spectral properties of the previous XMM-Newton observation of NGC 4579.

Table 4

Best-fit Gaussians to the Fe Complex

\begin{tabular}{|c|c|c|c|c|}
\hline Model & $\begin{array}{c}E \\
\mathrm{keV}\end{array}$ & $\begin{array}{c}\sigma \\
\mathrm{keV}\end{array}$ & $\begin{array}{c}N \\
\left(10^{-6} \text { photons }\right. \\
\left.\mathrm{cm}^{-2} \mathrm{~s}^{-1}\right)\end{array}$ & $\begin{array}{l}\text { EW } \\
\mathrm{eV}\end{array}$ \\
\hline Two Gaussians & $\begin{array}{l}6.4 \pm 0.1 \\
6.6 \pm 0.1\end{array}$ & $\begin{array}{c}0.018 \text { (f) } \\
0.5 \pm 0.1\end{array}$ & $\begin{array}{c}4 \pm 1 \\
19 \pm 3\end{array}$ & $\begin{array}{c}52_{-19}^{+13} \\
256_{-31}^{+36}\end{array}$ \\
\hline Three Gaussians & $\begin{array}{c}6.4(\mathrm{f}) \\
6.7(\mathrm{f}) \\
6.97(\mathrm{f})\end{array}$ & $\begin{array}{l}0.0(\mathrm{f}) \\
0.0(\mathrm{f}) \\
0.0(\mathrm{f})\end{array}$ & $\begin{array}{l}9 \pm 1 \\
4 \pm 1 \\
5 \pm 1\end{array}$ & $\begin{array}{c}118 \pm 14 \\
43 \pm 11 \\
72_{-14}^{+11}\end{array}$ \\
\hline
\end{tabular}

We also attempted to fit the NGC 4579 spectrum with the pexmon model. We removed the neutral Gaussian component from the model, which is self-consistently produced by pexmon in Compton-thick material. This fit results in a $\chi^{2}$ of 1975 for 1881 dof. Strong residuals are seen at high energies above $15 \mathrm{keV}$ where the model overestimates the flux from the source.
Given the possible Compton-thin origin of the neutral Fe line that we see in NGC 4579, we also fit the spectrum with the MYTorus model (Murphy \& Yaqoob 2009; Yaqoob 2012). This model includes the reprocessed continuum and neutral $\mathrm{Fe} \mathrm{K} \alpha, \mathrm{Fe} \mathrm{K} \beta$, and $\mathrm{Ni} \mathrm{K} \alpha$ emission by a torus-shaped structure with an opening angle of $60^{\circ}$. The continuum is assumed to be a PL with a high-energy cutoff fixed at $500 \mathrm{keV}$, and the column densities for the MYTorus tables reproducing the scattered continuum and emission lines are tied. A mekal component was also included to the continuum model. We also included contributions from narrow Fe XXV and Fe XXVI by fixing the two Gaussian components to their expected energies and their widths to 0 . The fit is good with a $\chi^{2}$ of 1919 for 1880 dof, and we do not observe any unmodeled residuals around the Fe complex. The hydrogen column density of the material responsible for the reprocessed continuum and the production of the neutral Fe K line is $N_{\mathrm{H}}=(7 \pm 2) \times 10^{22} \mathrm{~cm}^{-2}$ (errors quoted at the $3 \sigma$ level). We conclude that the line is likely originating from a Compton-thin material at large distances from the central $\mathrm{BH}$. 

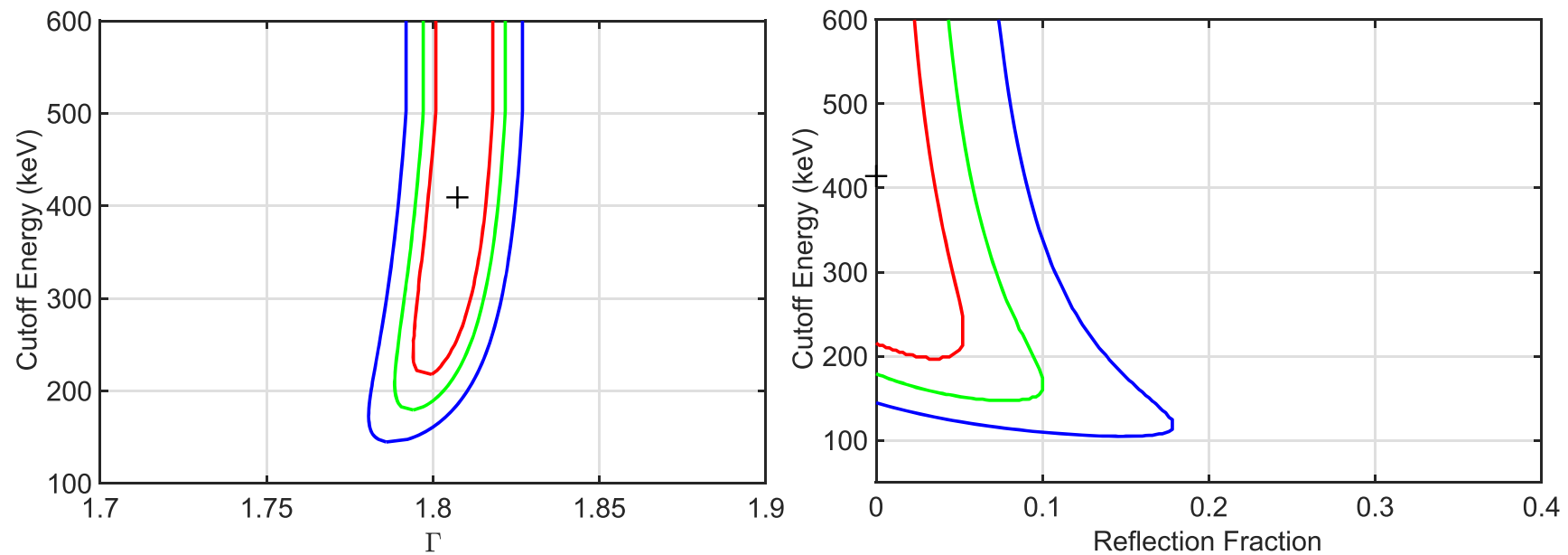

Figure 9. NGC $4579 E_{\text {cut }}-\Gamma$ contour plot (left panel) and $E_{\text {cut }}-R$ contour plot (right panel). Red, green, and blue show the $1 \sigma, 2 \sigma$, and $3 \sigma$ contours, respectively. See the text for more details.

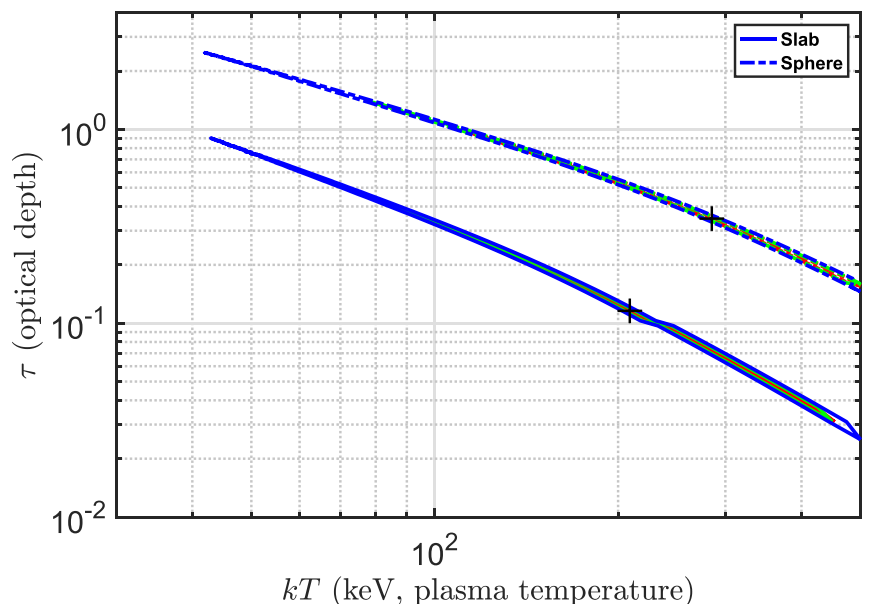

Figure 10. Contour plot of the plasma temperature and optical depth for the compTT model fit to the NGC 4579 data, assuming a slab geometry (solid lines) and spherical geometry (dashed lines); red, green, and blue show the $1 \sigma$, $2 \sigma$, and $3 \sigma$ contours, respectively.

Although we do not detect a cutoff in NGC 4579, we fit the broadband spectrum of the source with a physical Comptonization model, compTT in Xspec, to establish the allowed parameter space of the optical depth and temperature of the X-ray-emitting plasma, and to allow comparison with NGC 3998 and more broadly, luminous AGNs. We find a good fit to the spectrum with a $\chi^{2}$ of 1955 for 1880 dof, comparable to our best fit with a cutoff PL. We find a $1 \sigma$ lower limit on the plasma temperature of $\sim 170 \mathrm{keV}$ for either the slab or spherical geometry. We find an optical depth $\lesssim 1$ in both cases, indicating an optically thin plasma. The best-fit parameters are summarized in Table 3, and we show the contours $\tau$ versus $k T$ in Figure 10.

The $0.5-10 \mathrm{keV}$ flux that we derive in our observation is $60 \%$ larger than the one derived in the previous XMM-Newton observation of NGC 4579 (Dewangan et al. 2004). Hence, to search for any spectral variability in concordance to the brighter flux, we reanalyzed the historic XMM-Newton observation taken on 2004 June 30 (obs. ID 0112840101) as described in Section 2. We fit the spectra of the current and previous observations simultaneously with a continuum consistent with

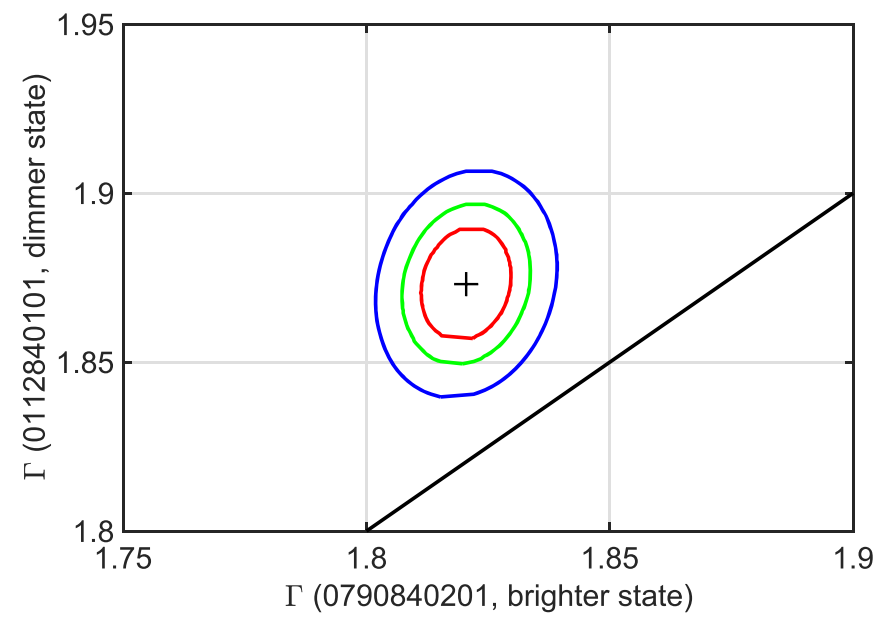

Figure 11. Contour plot of the photon indices derived from our simultaneous fit of the current XMM-Newton $+N u S T A R$ and previous XMM-Newton observations of NGC 4579. The solid line denotes the one-to-one relation. The brighter flux state of our current observation corresponds to the harder X-ray spectrum.

the one discussed above, i.e., a hot thermal plasma model and a cutoff PL (we fixed the cutoff energy for the previous $X M M$ Newton observation to $1 \mathrm{MeV}$ ). We linked the thermal spectral components between the two observations since no variability is expected in the hot diffuse gas over timescales of years. We let the cutoff PL index free to vary. First, we exclude the Fe complex between 5.5 and $8 \mathrm{keV}$. The residuals in this energy range compared to the best-fit continuum are shown in Figure 7. We fit the Fe complex with either two or three Gaussian components, in the same manner we conducted the spectral analysis of our current observation. We let the Gaussian parameters free to vary between the two observations. Within statistical uncertainties, we do not detect any variability in the $\mathrm{Fe}$ lines. Hence, we link the Gaussian parameters between the previous and the current observation. On the other hand, we detect a significant variability in the PL photon index, with the previous, dimmer observation possessing a softer spectrum. Figure 11 shows the contour plot of the indices from the two observations. We summarize in Table 3 the continuum spectral parameters of this fit. 


\section{Discussion}

The superior sensitivity of NUSTAR is allowing, for the first time, a detailed look at the hard X-ray view of LLAGNs, e.g., NGC 7213 and M81 (Ursini et al. 2015; Young et al. 2018). In this paper, we add to this growing sample by presenting the analysis of the broadband, $0.5-60 \mathrm{keV}, \mathrm{X}$-ray emission from two LLAGNs, NGC 3998 and NGC 4579, observed simultaneously with NUSTAR and XMM-Newton. The NUSTAR livetime exposures of the two sources are about 100 and $120 \mathrm{ks}$, respectively, taken over 2.5 and 3 days. This allowed us to look for variability at a timescale of days, which is rarely available for LLAGNs. We do not detect any strong variability in either source. Their power spectra in the $3-60 \mathrm{keV}$ band are consistent with white noise in the frequency range $\sim 10^{-5}$ $0.017 \mathrm{~Hz}$. This confirms that, indeed, most LLAGNs show little to no variability on timescales of days (Binder et al. 2009; Younes et al. 2011; Young et al. 2018), as opposed to the strong, short timescale variability seen in bright Seyfert galaxies (e.g., González-Martín \& Vaughan 2012).

The broadband $0.5-60 \mathrm{keV}$ continua of NGC 3998 and NGC 4579 are best fit with a cutoff PL (discussed in detail below) and a PL plus emission from hot thermally emitting plasma, respectively. Very small intrinsic absorption is required for NGC 3998, while for NGC 4579, the absorption column density is consistent with the Galactic one. This is in line with the picture of LLAGNs being devoid of absorbing material, such as a broad-line region and/or a torus in their central engine (Ho 2008). Moreover, She et al. (2018) showed a positive correlation between the absorption column density and the Eddington ratio, $\lambda_{\text {Edd }}$, in a sample of LLAGN, in striking contrast to luminous AGN (Ricci et al. 2017), but consistent with the expectation of absorption caused by outflowing material from a hot accretion flow (Yuan et al. 2015). Our two sources nicely follow the correlation, especially NGC 3998, where we indeed measure a very small intrinsic absorption corresponding to its $\lambda_{\mathrm{Edd}} \approx 10^{-5}$.

Neither source shows any hint of a Compton reflection hump at hard X-rays, a ubiquitous feature in luminous AGNs. The $3 \sigma$ upper limits that we derive on their reflection fractions, 0.30 and 0.18 for NGC 3998 and NGC 4579, respectively, are strongly constraining and place the two sources within the same $R$-Eddington ratio parameter space as other LLAGNs, e.g., M81 $(R<0.19$; Young et al. 2018), NGC $7231(R<0.20$; Ursini et al. 2015), and the low Eddington ratio AGN NGC 2110 ( $\left(\lambda_{\text {Edd }} \lesssim 10^{-2}, R<0.25\right.$; Marinucci et al. 2015). These very small reflection fractions are at odds with the much higher values measured for luminous AGNs (e.g., Risaliti et al. 2013; Parker et al. 2014; Kara et al. 2015a; Keck et al. 2015). With the addition of NGC 3998 and NGC 4579, there is now mounting evidence that the lack of reflection in the spectra of LLAGNs, if not universal, is a common feature of the population. The nature of the hard X-ray Compton hump in luminous AGNs is believed to be a reflection of the primary continuum source from the inner regions of a dense, optically thick accretion disk extending close to the innermost stable circular orbit (George \& Fabian 1991). The absence of this signature component from the spectra of LLAGNs reinforces the idea that their inner accretion disk is most likely truncated at a certain large radius from the $\mathrm{BH}$, which is instead filled with an optically thin, radiatively inefficient hot accretion flow (e.g., Esin et al. 1997; Narayan et al. 1997; Yuan 2007; Yuan \& Narayan 2014). Moreover, the absence of the Compton hump is consistent with the lack of another reflection component in LLAGNs, the broad $\mathrm{Fe} \mathrm{K} \alpha$ line (e.g., González-Martín et al. 2009; Younes et al. 2011). The latter is also ubiquitous in the $\mathrm{X}$-ray spectra of bright AGNs and have been shown to correlate temporally with the Compton hump, implying a similar physical nature (Kara et al. 2015b).

We confirm the existence of the Fe complex in NGC 4579, which was also detected with ASCA (Terashima et al. 1998, 2000) and the previous XMM-Newton observation of the source (Dewangan et al. 2004). The residuals in our present observation indicate a complex blending of lines, which requires at least two Gaussian components. If the energies and widths of the Gaussians are left free to vary, the residuals are best fit with an unresolved, narrow $\mathrm{Fe} \mathrm{K}$ line at $6.4 \mathrm{keV}$ and a moderately broad component consistent with highly ionized $\mathrm{Fe} \mathrm{XXV}$ at $\sim 6.7 \mathrm{keV}$. Nevertheless, the complex can also be well fit with three relatively narrow Gaussian components with energies fixed at $6.4,6.7$, and $6.97 \mathrm{keV}$, corresponding to $\mathrm{Fe} \mathrm{K}$ and highly ionized Fe XXV and Fe XXVI.

The narrow neutral component corresponding to $\mathrm{Fe} \mathrm{K} \alpha$ is unlikely to originate in Compton-thick material. Fitting the NGC 4579 spectrum with the reflection model pexmon, which self-consistently includes contribution from neutral Fe originating in Compton-thick material, does not adequately fit the data. We observe strong residuals at high energies above $20 \mathrm{keV}$, where the model overestimates the data. This result may imply that the formation of the observed Fe K line in Compton-thick material requires a strong reflection component at high energies that is not detected in the spectrum. On the other hand, the MYTorus model gives an adequate fit to the data, including the narrow Fe $\mathrm{K}$ feature. According to this model, the line is most likely produced in an optically thin region with a hydrogen column density $\sim 7 \times 10^{22} \mathrm{~cm}^{-2}$ (e.g., the broad-line region; Yaqoob \& Padmanabhan 2004). This is consistent with the presumed production site of the line in other LLAGNs, namely M81 and NGC 7213 (Ursini et al. 2015; Young et al. 2018). NGC 4579, similar to many other LLAGNs, show strong signatures of silicate emission features in the mid-IR ,strongly indicative of a dusty environment (Gallimore et al. 2010; Mason et al. 2012). Such environments are also possible sites for forming $\mathrm{Fe} \mathrm{K} \alpha$ lines; dust may help enhance the $\mathrm{Fe} \mathrm{K}$ equivalent width while suppressing strong high-energy reflection components due to a decrease in the backscattering opacity in the gas (Draine 2003), although to that end, we note that NGC 3998 does show strong silicate features with no indication of Fe K emission (Sturm et al. 2005; Mason et al. 2012).

The highly ionized Fe XXV and Fe XXVI lines are most likely the result of collisional ionization in optically thin gas, e.g., possibly at the transition layer between a truncated external thin accretion disk and a hot inner accretion flow. The temperature at the transition layer is supposedly $\sim 10^{7}-10^{9} \mathrm{~K}$ (Narayan et al. 1997; Perna et al. 2000), i.e., the temperatures required to produce highly ionized Fe. These lines can also be broad with equivalent width as large as a few hundred electron volts (Xu 2011). Such models have been shown to naturally explain the Fe XXV and Fe XXVI lines seen in M81 (Young et al. 2007) and the Fe XXV line seen in Sgr $\mathrm{A}^{*}$ (Xu et al. 2006; Wang et al. 2013). Another possible production site is the outflows from the accretion flow. In this scenario, blueshift corresponding to the outflow velocity is expected. Unfortunately, energy 
resolution and small number statistics render the distinction between the above two possibilities difficult.

The current observation of NGC 4579 shows a factor $\sim 60 \%$ larger flux compared to the previous XMM-Newton observation taken 13 years earlier. At the same time, the source spectrum shows a significant hardening (Figure 11). This is in line with the harder-when-brighter correlation established for different samples of LLAGNs (e.g., Younes et al. 2011; She et al. 2018). Assuming that the brighter flux is the result of increased accretion rate onto the $\mathrm{SMBH}$, the spectral hardening can be understood in the context of radiatively inefficient, optically thin, hot accretion flows. The increased densities in the flow will lead to more efficient inverse Compton scattering of primary synchrotron photons from the flow leading to a harder spectrum (e.g., Qiao \& Liu 2013; Yang et al. 2015). Within statistical uncertainties, we do not find any variability in the Fe-line complex between the two observations.

The NuSTAR+XMM-Newton data of NGC 3998 revealed the need for a high-energy cutoff to best fit its broadband $0.5-60 \mathrm{keV}$ spectrum. We measure a cutoff energy $E_{\text {cut }}=107_{-18}^{+27} \mathrm{keV}$. This represents the lowest and best constrained cutoff energy ever measured for an LLAGN. At first glance, this cutoff energy resembles the ones derived for Seyfert galaxies and other luminous AGNs. However, for normal AGNs, the cutoff energy is found to be inversely proportional to the Eddington ratio (Ricci et al. 2018; however, note that this relation is not seen when a much smaller catalog of bright AGNs observed with NUSTAR is considered; Tortosa et al. 2018). Sources with Eddington ratio $\lambda_{\mathrm{Edd}}=L_{\mathrm{bol}} / L_{\mathrm{Edd}}<0.1$ tend to have a cutoff energy of about $370 \mathrm{keV}$, while the ones with $\lambda_{\mathrm{Edd}}>0.1$ possess a much smaller cutoff energy, $E_{\text {cut }}=160 \mathrm{keV}$. NGC 3998, hence, represents an obvious outlier to this correlation, lying in the parameter space of low cutoff energy with a very small Eddington ratio.

The negative correlation as observed for normal AGNs could be understood in the context of a compact, X-ray-emitting corona (Haardt \& Maraschi 1993). The energy exchange becomes more efficient between photons and particles with decreasing size and/or increasing luminosity of the X-rayemitting corona, which cools down its plasma content, leading to a decrease in temperature. Following the definition of the compactness parameter $l \propto \lambda_{\text {Edd }} / R_{\mathrm{X}}$ (e.g., Fabian et al. 2015, 2017; Ricci et al. 2018) and assuming a typical luminous AGN corona size $R_{\mathrm{X}} \approx 10 R_{\mathrm{G}}$, where $R_{\mathrm{G}}$ is the gravitational radius, we estimate $l_{\text {NGC } 3998} \approx 0.01$. This can be thought of as a rough upper limit as $R_{\mathrm{X}}$ can indeed be larger for the LLAGN case. On the other hand, we find electron temperatures $\theta=k T_{\mathrm{e}} / m_{\mathrm{e}} c^{2}$ in the range of 0.03-0.3 from our Comptonization spectral fits. ${ }^{10}$ This small compactness parameter along with the low electron temperature places NGC 3998 in uncharted territory in the compactness-temperature plane (Fabian et al. 2015), closer to the electron-proton coupling line and bremsstrahlung as cooling modes rather than to electron-electron coupling. The latter is the preferred radiation process for luminous AGNs. This could imply that the physical properties of the X-ray-emitting region of NGC 3998, and possibly most LLAGNs, differ markedly from luminous AGNs. The very small compactness parameter and radiation process argue for the expectation of a large, optically thin hot accretion flow.

\footnotetext{
${ }^{10}$ The electron temperatures that we derive through the Comptonization model do indeed follow the relation $k T_{\mathrm{e}}=E_{\text {cut }} / 2$ for optically thin plasma, where $E_{\text {cut }}$ is the cutoff energy established through our cutoff PL modeling.
}

We note that using the lower limits that we derive from our Comptonization spectral fits to the NGC 4579 spectra, we derive an electron temperature $\theta \gtrsim 0.1$ and a compactness parameter $\lesssim 0.1$. These limits fall within the range of the electron-proton coupling line. However, because only upper limits can be derived in such a case, the spectrum could still be consistent with electron-electron coupling, similar to what is observed for bright AGNs. Future soft $\mathrm{MeV}$ missions are crucial to constrain the high-energy cutoff in LLAGN spectra and better understand the physical properties of the high-energy emitting region in these sources.

The high-quality, simultaneous NuSTAR+XMM-Newton data allowed us to fit physically motivated models to the broadband spectrum of NGC 3998. A bremsstrahlung-only model does not give an adequate fit to the data. In fact, we also tried to fit the spectrum with two bremsstrahlung components, akin to emission from a two-temperature hot accretion flow, and this too resulted in a statistically poor fit. The Comptonization model compTT, on the other hand, resulted in a good fit, with best-fit statistics equivalent to the one with the phenomenological cutoff PL model. Both a spherical geometry for the Compton cloud and a slab geometry resulted in equally good fits. The contour plots of these fits, as shown in Figure 6, indicate a high level of degeneracy between the electron temperature and the optical depth, a fact already known for Comptonization models (e.g., Brenneman et al. 2014; Baloković et al. 2015). Nonetheless, within the $3 \sigma$ level, both geometries result in a plasma temperature ranging from 15 to $150 \mathrm{keV}$. The optical depth, however, differs between the two geometries and ranges between 0.8 and 6 for the spherical geometry and $0.2-2.5$ for the slab geometry. Smaller optical depths for the slab geometry compared to the spherical case have already been pointed out (in, e.g., Lubiński et al. 2010; Brenneman et al. 2014; Baloković et al. 2015) and are the result of integration over radial distances and scale heights, respectively. These results support the picture of Comptonization as the dominant emission process over bremsstrahlung in NGC 3998. Our results are also consistent with Comptonization emission from optically thin hot accretion flows (e.g., ADAF, Yuan 2007), which predict high temperatures of $\sim 100 \mathrm{keV}$ and small optical depths $\tau \lesssim 1$ (derived after integrating vertically, hence, to be compared with the slab geometry results). Finally, this low-energy cutoff detection in NGC 3998 limits the validity range of jet emission models. Pure synchrotron emission from a jet has inherent difficulty producing a cutoff at hard X-rays (Zdziarski et al. 2004); nevertheless, Comptonization in the base of a jet has been shown to produce such curvature in the X-ray spectra of hard-state X-ray binaries (e.g., Markoff et al. 2005).

\section{Summary}

In the present work, we report on the broadband X-ray timing and spectral analysis of the two LLAGNs NGC 3998 and NGC 4579 through simultaneous NuSTAR + XMM-Newton observations. The summary of our main results is as follows:

1. We do not detect any significant variability in either source over the $\sim 3$ day length of the NUSTAR observations; both sources have power spectra consisting of white noise in the frequency range $\sim 10^{-5}-0.017 \mathrm{~Hz}$.

2. The broadband $0.5-60 \mathrm{keV}$ spectrum of NGC 3998 is best fit with a cutoff power law and cutoff energy $E_{\text {cut }}=$ $107_{-18}^{+27} \mathrm{keV}$. This represents the lowest and best 
constrained high-energy cutoff ever measured for an LLAGN. Such a relatively low value places NGC 3998 as an outlier to the anticorrelation found in luminous AGNs between $E_{\text {cut }}$ and the Eddington ratio.

3. The NGC 3998 spectrum is consistent with a Comptonization model with either a sphere or slab geometry with optical depths in the range of $0.8-6$ and $0.2-2.5$, respectively, corresponding to plasma temperatures between 20 and $150 \mathrm{keV}$. Its spectrum is inconsistent with bremsstrahlung emission.

4. The broadband $0.5-60 \mathrm{keV}$ spectrum of NGC 4579 is best fit with a combination of a hot thermal plasma model, a power law, and a blend of Gaussians to fit an $\mathrm{Fe}$ complex observed between 6 and $7 \mathrm{keV}$. These residuals could either be fit with a narrow Fe $\mathrm{K}$ line at $6.4 \mathrm{keV}$ and a moderately broad Fe XXV line, or with three relatively narrow lines, which include contribution from Fe XXVI.

5. The NGC 4579 flux is $60 \%$ brighter than previously detected with XMM-Newton and is accompanied by a hardening in the spectrum.

6. Neither source shows any reflection hump with $3 \sigma$ reflection fraction upper limits $R<0.3$ and $R<0.18$ for NGC 3998 and NGC 4579, respectively.

The very low reflection fractions that we derive for our two sources, along with the lack of variability and broad Fe $\mathrm{K} \alpha$ lines, argue for an altered accretion geometry in LLAGNs compared to luminous AGNs. This reinforces the picture of a truncated thin disk that is replaced by a pressure-dominated hot accretion flow. This picture is also in line with our finding of a harder-when-brighter spectrum for NGC 4579. Finally, our most interesting result, the relatively low high-energy cutoff that we measure for NGC $3998\left(E_{\text {cut }}=107_{-18}^{+27} \mathrm{keV}\right)$, is inconsistent with the picture of X-ray emission emanating from a compact corona regulated through electron-electron coupling, as is widely accepted for luminous AGNs. The combination of very low compactness and 20-150 keV plasma temperature that we derive is more in line with emission from a large optically thin volume where electron-proton coupling and bremsstrahlung act as cooling modes. Such picture is again in favor of emission from an optically thin hot accretion flow.

This work made use of data from the NuSTAR mission, a project led by the California Institute of Technology, managed by the Jet Propulsion Laboratory, and funded by the National Aeronautics and Space Administration. We thank the NuSTAR Operations, Software and Calibration teams for support with the execution and analysis of these observations. This research has made use of the NuSTAR Data Analysis Software (NuSTARDAS) jointly developed by the ASI Science Data Center (ASDC, Italy) and the California Institute of Technology (USA). G.Y. acknowledges support from NASA under NuSTAR Guest Observer cycle-2, proposal number 15-NUSTAR215-0024. L.C.H. was supported by the National Key R\&D Program of China (2016YFA0400702) and the National Science Foundation of China (11473002, 11721303). Y.T. is supported by JSPS Grants-in-Aid for Scientific Research $15 \mathrm{H} 02070$ and 16K05296. F.G.X. and F.Y. are supported in part by National Key R\&D Program of China (grants 2016YFA0400804 and 2016YFA0400704), the Natural Science Foundation of China (grants 11873074, 11573051, 11633006, $11650110427,11661161012,11303008$, and 11473002), and the Key Research Program of Frontier Sciences of CAS (grants QYZDJSSW-SYS008 and QYZDB-SSW-SYS033). F.G.X. is also supported by the Youth Innovation Promotion Association of CAS (ID. 2016243) and the Natural Science Foundation of Shanghai (No. 17ZR1435800). We thank the referee for a concise and thorough reading of the manuscript that led to an improved version of the article.

\section{ORCID iDs}

George Younes (iD https://orcid.org/0000-0002-7991-028X

Andrew Ptak (i) https://orcid.org/0000-0001-5655-1440

Luis C. Ho (1) https://orcid.org/0000-0001-6947-5846

Feng Yuan (10) https://orcid.org/0000-0003-3564-6437

Daniela Huppenkothen (i) https://orcid.org/0000-00021169-7486

\section{References}

Arnaud, K. A. 1996, in ASP Conf. Ser. 101, Astronomical Data Analysis Software and Systems V, ed. G. H. Jacoby \& J. Barnes (San Francisco, CA: ASP), 17

Baloković, M., Matt, G., Harrison, F. A., et al. 2015, ApJ, 800, 62

Binder, B., Markowitz, A., \& Rothschild, R. E. 2009, ApJ, 691, 431

Blandford, R. D., \& Begelman, M. C. 1999, MNRAS, 303, L1

Brenneman, L. W., Madejski, G., Fuerst, F., et al. 2014, ApJ, 788, 61

Dewangan, G. C., Griffiths, R. E., Di Matteo, T., \& Schurch, N. J. 2004, ApJ, 607, 788

Di Matteo, T., Carilli, C. L., \& Fabian, A. C. 2001, ApJ, 547, 731

Draine, B. T. 2003, ApJ, 598, 1026

Eracleous, M., Hwang, J. A., \& Flohic, H. M. L. G. 2010, ApJS, 187, 135

Esin, A. A., McClintock, J. E., \& Narayan, R. 1997, ApJ, 489, 865

Fabbiano, G. 1989, ARA\&A, 27, 87

Fabian, A. C., Lohfink, A., Belmont, R., Malzac, J., \& Coppi, P. 2017, MNRAS, 467, 2566

Fabian, A. C., Lohfink, A., Kara, E., et al. 2015, MNRAS, 451, 4375

Flohic, H. M. L. G., Eracleous, M., Chartas, G., Shields, J. C., \& Moran, E. C. 2006, ApJ, 647, 140

Gallimore, J. F., Yzaguirre, A., Jakoboski, J., et al. 2010, ApJS, 187, 172

George, I. M., \& Fabian, A. C. 1991, MNRAS, 249, 352

González-Martín, O., Masegosa, J., Márquez, I., Guainazzi, M., \& Jiménez-Bailón, E. 2009, A\&A, 506, 1107

González-Martín, O., \& Vaughan, S. 2012, A\&A, 544, A80

Gu, M., \& Cao, X. 2009, MNRAS, 399, 349

Haardt, F., \& Maraschi, L. 1993, ApJ, 413, 507

Harrison, F. A., Craig, W. W., Christensen, F. E., et al. 2013, ApJ, 770, 103

Hernández-García, L., González-Martín, O., Masegosa, J., \& Márquez, I. 2014, A\&A, 569, A26

Ho, L. C. 1999, ApJ, 516, 672

Ho, L. C. 2008, ARA\&A, 46, 475

Ho, L. C. 2009, ApJ, 699, 626

Ho, L. C., Filippenko, A. V., \& Sargent, W. L. W. 1997, ApJ, 487, 568

Ho, L. C., Filippenko, A. V., Sargent, W. L. W., \& Peng, C. Y. 1997, ApJS, 112,391

Ho, L. C., Greene, J. E., Filippenko, A. V., \& Sargent, W. L. W. 2009, ApJS, 183,1

Kaastra, J. S., \& Mewe, R. 1993, A\&AS, 97, 443

Kara, E., Fabian, A. C., Lohfink, A. M., et al. 2015a, MNRAS, 449, 234

Kara, E., Zoghbi, A., Marinucci, A., et al. 2015b, MNRAS, 446, 737

Keck, M. L., Brenneman, L. W., Ballantyne, D. R., et al. 2015, ApJ, 806, 149

Kormendy, J., \& Ho, L. C. 2013, ARA\&A, 51, 511

Lubiński, P., Zdziarski, A. A., Walter, R., et al. 2010, MNRAS, 408, 1851

Magdziarz, P., \& Zdziarski, A. A. 1995, MNRAS, 273, 837

Mantovani, G., Nandra, K., \& Ponti, G. 2016, MNRAS, 458, 4198

Marinucci, A., Matt, G., Bianchi, S., et al. 2015, MNRAS, 447, 160

Markoff, S., Nowak, M. A., \& Wilms, J. 2005, ApJ, 635, 1203

Mason, R. E., Lopez-Rodriguez, E., Packham, C., et al. 2012, AJ, 144, 11

Mewe, R., Gronenschild, E. H. B. M., \& van den Oord, G. H. J. 1985, A\&AS, 62, 197

Murphy, K. D., \& Yaqoob, T. 2009, MNRAS, 397, 1549

Nagar, N. M., Falcke, H., \& Wilson, A. S. 2005, A\&A, 435, 521

Nandra, K., O’Neill, P. M., George, I. M., \& Reeves, J. N. 2007, MNRAS, 382, 194

Narayan, R., Garcia, M. R., \& McClintock, J. E. 1997, ApJL, 478, L79

Narayan, R., Igumenshchev, I. V., \& Abramowicz, M. A. 2000, ApJ, 539, 798 
Narayan, R., \& McClintock, J. E. 2008, NewAR, 51, 733

Narayan, R., \& Yi, I. 1994, ApJL, 428, L13

Nemmen, R. S., Storchi-Bergmann, T., \& Eracleous, M. 2014, MNRAS, 438, 2804

Parker, M. L., Wilkins, D. R., Fabian, A. C., et al. 2014, MNRAS, 443, 1723

Perna, R., Raymond, J., \& Narayan, R. 2000, ApJ, 541, 898

Pian, E., Romano, P., Maoz, D., et al. 2010, MNRAS, 401, 677

Ptak, A., Terashima, Y., Ho, L. C., \& Quataert, E. 2004, ApJ, 606, 173

Ptak, A., Yaqoob, T., Mushotzky, R., Serlemitsos, P., \& Griffiths, R. 1998, ApJL, 501, L37

Qiao, E., \& Liu, B. F. 2013, ApJ, 764, 2

Quataert, E., Di Matteo, T., Narayan, R., \& Ho, L. C. 1999, ApJL, 525, L89

Ricci, C., Ho, L. C., Fabian, A. C., et al. 2018, MNRAS, 480, 1819

Ricci, C., Trakhtenbrot, B., Koss, M. J., et al. 2017, Natur, 549, 488

Risaliti, G., Harrison, F. A., Madsen, K. K., et al. 2013, Natur, 494, 449

Shakura, N. I., \& Sunyaev, R. A. 1973, A\&A, 24, 337

She, R., Ho, L. C., Feng, H., \& Cui, C. 2018, ApJ, 859, 152

Sobolewska, M. A., \& Papadakis, I. E. 2009, MNRAS, 399, 1597

Springob, C. M., Masters, K. L., Haynes, M. P., Giovanelli, R., \& Marinoni, C. 2007, ApJS, 172, 599

Strüder, L., Briel, U., Dennerl, K., et al. 2001, A\&A, 365, L18

Sturm, E., Schweitzer, M., Lutz, D., et al. 2005, ApJL, 629, L21

Terashima, Y., Ho, L. C., Ptak, A. F., et al. 2000, ApJL, 535, L79

Terashima, Y., Kunieda, H., Misaki, K., et al. 1998, ApJ, 503, 212

Titarchuk, L. 1994, ApJ, 434, 570

Tonry, J. L., Dressler, A., Blakeslee, J. P., et al. 2001, ApJ, 546, 681

Tortosa, A., Bianchi, S., Marinucci, A., Matt, G., \& Petrucci, P. O. 2018, A\&A, 614, A37

Ursini, F., Marinucci, A., Matt, G., et al. 2015, MNRAS, 452, 3266
Verner, D. A., Ferland, G. J., Korista, K. T., \& Yakovlev, D. G. 1996, ApJ, 465,487

Wang, Q. D., Nowak, M. A., Markoff, S. B., et al. 2013, Sci, 341, 981

Wilms, J., Allen, A., \& McCray, R. 2000, ApJ, 542, 914

Xu, Y.-D. 2011, ApJ, 729, 10

Xu, Y.-D., \& Cao, X.-W. 2009, RAA, 9, 401

Xu, Y.-D., Narayan, R., Quataert, E., Yuan, F., \& Baganoff, F. K. 2006, ApJ, 640,319

Yang, Q.-X., Xie, F.-G., Yuan, F., et al. 2015, MNRAS, 447, 1692

Yaqoob, T. 2012, MNRAS, 423, 3360

Yaqoob, T., \& Padmanabhan, U. 2004, ApJ, 604, 63

Younes, G., Porquet, D., Sabra, B., et al. 2010, A\&A, 517, A33

Younes, G., Porquet, D., Sabra, B., \& Reeves, J. N. 2011, A\&A, 530, A149

Younes, G., Porquet, D., Sabra, B., Reeves, J. N., \& Grosso, N. 2012, A\&A, 539, A104

Young, A. J., McHardy, I., Emmanoulopoulos, D., \& Connolly, S. 2018, MNRAS, 476, 5698

Young, A. J., Nowak, M. A., Markoff, S., Marshall, H. L., \& Canizares, C. R. 2007, ApJ, 669, 830

Yuan, F. 2007, in ASP Conf. Ser. 373, The Central Engine of Active Galactic Nuclei, ed. L. C. Ho \& J.-W. Wang (San Francisco, CA: ASP), 95

Yuan, F., Gan, Z., Narayan, R., et al. 2015, ApJ, 804, 101

Yuan, F., \& Narayan, R. 2014, ARA\&A, 52, 529

Yuan, F., Quataert, E., \& Narayan, R. 2004, ApJ, 606, 894

Zdziarski, A. A., Gierliński, M., Mikołajewska, J., et al. 2004, MNRAS, 351,791

Zhang, W. M., Soria, R., Zhang, S. N., Swartz, D. A., \& Liu, J. F. 2009, ApJ, 699,281 